\title{
Physiological predictors of exertional oxygen desaturation in patients with fibrotic interstitial lung disease
}

To the Editor:

In patients with fibrotic interstitial lung disease (ILD), hypoxaemia on exertion is frequent, and contributes to exercise intolerance, exertional dyspnoea and reduced quality of life [1-3]. Clinically significant exertional hypoxaemia is typically defined as a drop in transcutaneous arterial oxygen saturation $\left(S_{\mathrm{pO}_{2}}\right)$ to $\leqslant 88 \%$ on a 6 -min walk test $(6 \mathrm{MWT})[4]$, and is associated with reduced survival in ILD patients [5].

In the AmbOx trial, ambulatory oxygen was associated with improved health-related quality of life in ILD patients with isolated exertional hypoxaemia [6]. Although exertional desaturation has been correlated with gas transfer measurements $[7,8]$, few data are available on the predictors of a drop of $S_{\mathrm{pO}_{2}}$ to $\leqslant 88 \%$ in ILD, and no physiological parameter thresholds have been identified to help select ILD patients who would most benefit from performing a 6MWT.

We analysed predictors of oxygen desaturation $\left(S_{\mathrm{pO}_{2}} \leqslant 88 \%\right)$ on $6 \mathrm{MWT}$ in ILD patients without severe resting hypoxia $\left(S_{\mathrm{pO}_{2}}\right.$ at rest $\left.\geqslant 92 \%\right)$ in a derivation cohort $(\mathrm{n}=146$; patients screened at the Royal Brompton Hospital (RBH; London, UK) for the AmbOx study between September 2014 and July 2016) [6] and a validation cohort ( $\mathrm{n}=154$; consecutive ILD referrals to RBH seen between August 2016 and May 2018). Approval for this study was obtained from the $\mathrm{RBH}$ institutional ethics committee. Patients requiring oxygen at rest and/or with clinical signs of right heart failure and/or symptomatic ischaemic heart disease were excluded. Lung function tests including forced vital capacity (FVC), forced expiratory volume $\left(\mathrm{FEV}_{1}\right)$ and diffusing capacity of the lung for carbon monoxide $\left(D_{\mathrm{LCO}}\right)$ were performed in all patients within 6 months of the $6 \mathrm{MWT}$. The composite physiological index (CPI) was used as a functional index of lung fibrosis severity [9]. The 6MWT was performed as described previously [10]. Categorical variables were compared using the Chi-squared test or the Fisher exact test and continuous variables with t-test or nonparametric Mann-Whitney test, as appropriate. Univariable logistic regression analysis was used to identify variables predictive of 6MWT oxygen desaturation. Any factors potentially associated on univariable analysis with $S_{\mathrm{pO}_{2}} \leqslant 88 \%$ on $6 \mathrm{MWT}(\mathrm{p}<0.10)$ were added to the multivariable model. If two variables were highly correlated ( $\mathrm{r}$ coefficient $>| \pm 0.30|$ ), the one with the largest variance was excluded from the multivariable analysis [11]. Finally, a backward stepwise selection ( $\mathrm{p}$-in $<0.05$, p-out $>0.10)$ was used to determine the factors independently associated with $S_{\mathrm{pO}_{2}} \leqslant 88 \%$ on $6 \mathrm{MWT}$. Receiver operating characteristic analyses were performed on significant variables derived from the final logistic regression models, and optimal cut-off points for each variable were identified by using Youden's index. The Hosmer-Lemeshow goodness-of-fit test was performed to assess the overall fit of the final models. All statistical analyses were performed using IBM SPSS (version 25.0; IBM, Armonk, NY, USA). A p-value of $<0.05$ was considered statistically significant.

A total of 300 ILD patients (derivation cohort $n=146$, validation cohort $n=154$ ) were included in the analysis. Age, sex and smoking history did not differ significantly between the two cohorts (derivation cohort: age $66.5 \pm 10.4$ years, male $65 \%$, ever-smokers $55.5 \%$; validation cohort: age $65.2 \pm 10.7$ years, male $61 \%$, ever-smokers 55.8\%). Overall, $112(37.3 \%)$ patients had a multidisciplinary team diagnosis of IPF, 65 (21.6\%) chronic hypersensitivity pneumonitis, 35 (11.7\%) connective tissue disease-associated ILD, 14

@ERSpublications

In patients with interstitial lung disease, exertional hypoxaemia has quality of life and prognostic implications. A simple "DeOX" score predictive of exertional oxygen desaturation $\left(\mathrm{P}_{\mathrm{pO}_{2}} \leqslant 88 \%\right.$ on 6MWT) is proposed, using $S_{\mathrm{pO}_{2}}$ at rest and $D_{\mathrm{LCO}}$. http://bit.ly/36ytigE

Cite this article as: Alfieri V, Crisafulli E, Visca D, et al. Physiological predictors of exertional oxygen desaturation in patients with fibrotic interstitial lung disease. Eur Respir J 2020; 55: 1901681 [https://doi. org/10.1183/13993003.01681-2019]. 
(4.7\%) nonspecific interstitial pneumonia, eight (2.7\%) sarcoidosis and 66 (22\%) other ILDs. Patients from the validation cohort had less-severe disease (mean \pm SD CPI $44.4 \pm 13$ versus $52.9 \pm 10.6, \mathrm{p}=0.0001$ ) and were less likely to desaturate on 6MWT (26\% versus 63\%, p<0.001). For the 297 patients with available computed tomography scans (derivation cohort $n=144$, validation cohort $n=153$ ), emphysema was scored as absent (emphysema score $=0$ ), limited (visible in the upper areas of the lung but not reaching the carina; emphysema score $=1$ ) and extensive (reaching the carina or further caudally; emphysema score $=2$ ). Limited emphysema was present in $23(16 \%)$ and $12(7.8 \%)$ patients in the derivation and validation cohorts, respectively, while extensive emphysema was present in five (3.5\%) and six (3.9\%) patients, respectively.

On univariable analysis, variables associated with desaturation on 6MWT in the derivation cohort included $S_{\mathrm{pO}_{2}}$ at rest (OR 0.57, 95\% CI 0.45-0.73), $D_{\mathrm{LCO}} \%$ (OR 0.94, 95\% CI 0.91-0.97), FVC \% (OR 0.97, 95\% CI 0.96-0.99), $\mathrm{FEV}_{1} \%$ (OR 0.98, 95\% CI 0.96-0.99) and CPI (OR 1.06, 95\% CI 1.02-1.09), and were confirmed in the validation cohort (data not shown), while diagnosis of idiopathic pulmonary fibrosis (IPF), age, sex, body mass index and smoking history were not associated in either cohort. On multivariable analysis, only $D_{\mathrm{LCO}}$ and $\mathrm{S}_{\mathrm{pO}_{2}}$ remained independently predictive of oxygen desaturation on $6 \mathrm{MWT} \leqslant 88 \%$ in each cohort, with adjustment for age, sex, smoking history and either $S_{\mathrm{pO}_{2}}$ or $D_{\mathrm{LCO}}$ as appropriate (adjusted (a) odds ratios for $D_{\mathrm{LCO}}$ in derivation cohort aOR 0.94, 95\% CI 0.90-0.98, p=0.002 and validation cohort aOR $0.91,95 \%$ CI $0.87-0.95, \mathrm{p}<0.0001$; for resting $S_{\mathrm{pO}_{2}}$ in derivation cohort aOR $0.56,95 \%$ CI $0.43-0.73, \mathrm{p}<0.0001$ and validation cohort aOR $0.57,95 \%$ CI $0.43-0.74, \mathrm{p}<0.0001)$. The optimal predictive thresholds for $D_{\mathrm{LCO}}(\leqslant 40 \%)$ and $S_{\mathrm{pO}_{2}}(\leqslant 95 \%)$ were identified in the derivation cohort and confirmed in the validation cohort. The two variables were then integrated into a predictive "DeOX" score $\left(0-2 ; 0=S_{\mathrm{pO}_{2}}>95 \%\right.$ and $D_{\mathrm{LCO}}>40 \% ; 1=S_{\mathrm{pO}_{2}} \leqslant 95 \%$ or $D_{\mathrm{LCO}} \leqslant 40 \% ; 2=D_{\mathrm{LCO}} \leqslant 40 \%$ and $\left.S_{\mathrm{pO}_{2}} \leqslant 95 \%\right)$. The presence of one or both variables progressively increases the risk of desaturation in both cohorts,

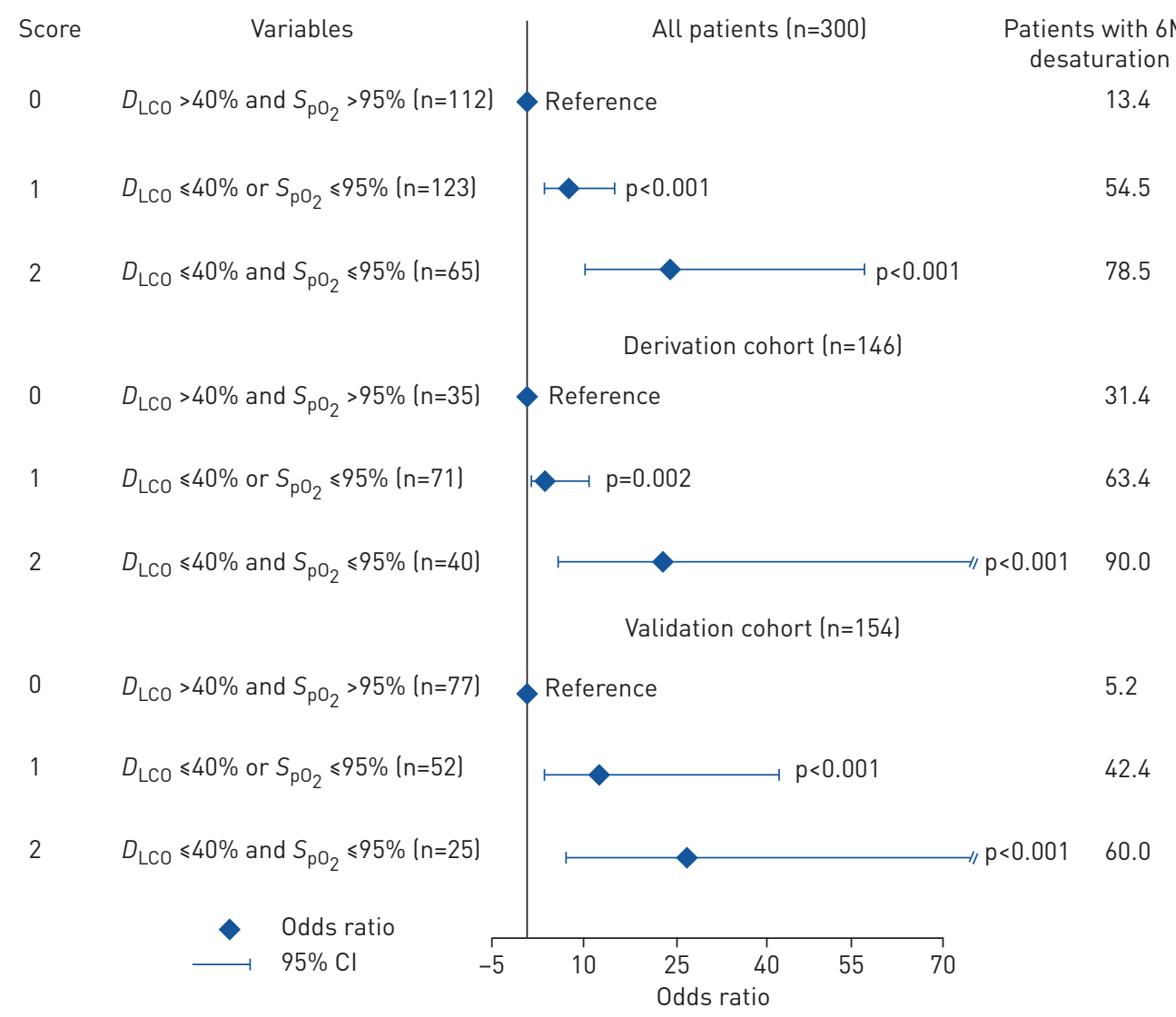

FIGURE 1 Odds ratio for desaturation (transcutaneous arterial oxygen saturation $\left(S_{\mathrm{po}_{2}}\right) \leqslant 88 \%$ ) on 6 -min walk test (6MWT), according to presence of neither (score=0), one (score=1) or both (score=2) thresholds (diffusing capacity of the lung for carbon monoxide $\left.\left(D_{\mathrm{LCO}}\right) \leqslant 40 \% ; \mathrm{S}_{\mathrm{pO}_{2}} \leqslant 95 \%\right)$. All patients: OR 8.1 (95\% Cl 4.14-15.88) for score=1, and $24.8(95 \% \mathrm{Cl} 11.78-57.04)$ for score=2. Derivation cohort: OR 4.5 (95\% Cl 1.77-11.53) for score=1, and 23.9 (95\% Cl 6.37-89.86) for score=2. Validation cohort: OR 13.4 (95\% Cl 4.19-42.87) for score=1, and 27.3 $195 \% \mathrm{Cl} 7.31-101.57)$ for score=2. All odds ratios are adjusted for anthropometric characteristics. HosmerLemeshow goodness-of-fit tests $p=0.995, p=0.92$ and $p=0.56$ for multivariate adjusted models in all patients, derivation and validation cohorts, respectively. 
separately and in combination (figure 1). Considering both cohorts together, our data show that with a DeOX score of 1 , the odds ratio for 6MWT desaturation was 8.1 (95\% CI 4.14-15.88) with a likelihood ratio of 1.5 (95\% CI 1.1-1.4), and increased markedly with a score of 2 (OR 24.8, 95\% CI 11.78-57.04) with a likelihood ratio of 4.4 (95\% CI 2.6-3.5). The strength of this association did not change on adjusting for the presence of the emphysema score in the multivariable analysis in each cohort separately, or in both combined (for both cohorts OR 8.7, 95\% CI 4.4-17.3 for a DeOX score of 1, and OR 25.7, 95\% CI 11.1-59.1 for a score of 2). Of note, $78.5 \%$ of patients with a DeOX score of 2 desaturated on a $6 \mathrm{MWT}$, highlighting the cohort of ILD patients for whom more efforts should be made to ensure that oxygen desaturation on exercise is promptly tested.

To our knowledge, this is the largest study correlating physiological variables with the likelihood of oxygen desaturation on 6MWT in fibrotic ILD patients. Our novel predictive score combines $D_{\mathrm{LCO}}$ and $S_{\mathrm{pO}_{2}}$ at rest; two non-invasive variables readily obtainable in a respiratory service. The limited time and staff available in busy outpatient services mean that the $6 \mathrm{MWT}$ is not consistently performed on routine follow-up, and the identification of exertional hypoxia may be missed. The finding that $D_{\mathrm{LCO}}$ and $S_{\mathrm{pO}_{2}}$ are independent determinants of oxygen desaturation on exercise suggests that two separate phenomena are being captured. While in $D_{\mathrm{LCO}}$ we have our best measure of morphological disease severity, a lower $S_{\mathrm{pO}_{2}}$ for a given $D_{\mathrm{LCO}}$ is likely to be linked to an important pulmonary vascular component $[12,13]$.

Our study has a number of limitations, including its retrospective nature. However, the predictive ability of the identified thresholds was observed in two independent cohorts, improving the confidence in our findings. The two cohorts differed in severity, with a significantly higher proportion of patients desaturating on 6MWT in the derivation compared to the validation cohort. The derivation cohort comprised patients screened for the AmbOx trial. Clinicians referring patients for a trial of supplemental oxygen will have been more likely to select those with more extensive ILD and/or reporting significant breathlessness on exertion. There was no selection bias for the validation cohort, where all consecutive patients not requiring oxygen at rest and meeting our exclusion criteria were included. Of interest, the score worked best in this less severe "unselected" validation cohort, more likely to be representative of the ILD population attending respiratory services. All patients were seen in the same centre with a centralised lung function lab. As $D_{\mathrm{LCO}}$ measurements can be subject to interlaboratory variation, the findings should be confirmed in different centres, in order to confirm the robustness of the score across different lung function facilities. Finally, as the $6 \mathrm{MWT}$ was performed at sea level, the validity of the score should be tested at different altitudes.

In conclusion, our data suggest that resting $S_{\mathrm{pO}_{2}}$ and $D_{\mathrm{LCO}}$ percentage are independently correlated with significant desaturation on a $6 \mathrm{MWT}$. We propose a novel simple scoring system to predict the risk of oxygen desaturation during 6MWT in patients with ILD, using a combination of two easily obtainable non-invasive physiological variables. We suggest that if confirmed by different centres, this score could be useful in clinical practice to screen for ILD patients likely to benefit from a formal 6MWT.

Veronica Alfieri ${ }^{1,2}$, Ernesto Crisafulli ${ }^{1,3}$, Dina Visca $\circledast^{2,4}$, Wing Ho Chong ${ }^{5,6}$, Carmel Stock ${ }^{2,7}$, Letizia Mori ${ }^{8}$, Angelo de Lauretis,, Vicky Tsipouri ${ }^{2}$, Felix Chua $^{2}$, Vasilis Kouranos ${ }^{2}$, Maria Kokosi ${ }^{2}$, Charlotte Hogben ${ }^{2}$, Philip L. Molyneaux $\oplus^{2,7}$, Peter M. George ${ }^{2,7}$, Toby M. Maher ${ }^{2,7}$, Alfredo A. Chetta ${ }^{1}$, Piersante Sestini ${ }^{10}$, Athol U. Wells ${ }^{2,7}$ and Elisabetta A Renzoni ${ }^{2,7}$

${ }^{1}$ Dept of Medicine and Surgery, Respiratory Disease and Lung Function Unit, University of Parma, Parma, Italy. ${ }^{2}$ Interstitial Lung Disease Unit, Royal Brompton Hospital, London, UK. ${ }^{3}$ Dept of Medicine, Respiratory Medicine Unit, University of Verona, Verona, Italy. ${ }^{4}$ Division of Pulmonary Rehabilitation, Istituti Clinici Scientifici Maugeri, IRCCS Tradate, Italy. ${ }^{5}$ Dept of Radiology, Tuen Mun Hospital, Hong Kong SAR. ${ }^{6}$ Dept of Radiology, Royal Brompton Hospital, London, UK. ${ }^{7}$ National Heart and Lung Institute, Imperial College, London, UK. ${ }^{8}$ Respiratory Medicine, Northwick Park Hospital, Harrow, UK. ${ }^{9}$ Unità Operativa Complessa di Pneumologia, ASST-Rhodese, Garbagnate Milanese, Italy. ${ }^{10}$ Dept of Medicine, Surgery and Neuroscience, University of Siena, Siena, Italy.

Correspondence: Veronica Alfieri, Dept of Medicine and Surgery, Respiratory Disease and Lung Function Unit, University of Parma, Via Gramsci 14 - 43126, Parma, Italy. E-mail: veronica.alfieri@unipr.it

Received: 28 Aug 2019 | Accepted after revision: 25 Oct 2019

Conflict of interest: V. Alfieri has nothing to disclose. E. Crisafulli has nothing to disclose. D. Visca has nothing to disclose. W.H. Chong has nothing to disclose. C. Stock has nothing to disclose. L. Mori has nothing to disclose. A. de Lauretis has nothing to disclose. V. Tsipouri has nothing to disclose. F. Chua reports lecture fees and advisory board fees from Boehringer Ingelheim and from Roche, outside the submitted work. V. Kouranos has nothing to disclose. M. Kokosi has nothing to disclose. C. Hogben has nothing to disclose. P.L. Molyneaux has, via his institution, received industry-academic funding from Roche, Boehringer Ingelheim and Galapagos and has received speaker fees from Roche. P.M. George reports grants, personal fees and non-financial support from Boehringer Ingelheim, personal fees and non-financial support from Roche, personal fees from Teva, outside the submitted work. T.M. Maher has, via his institution, received industry-academic funding from GlaxoSmithKline R\&D and UCB and has received consultancy or speaker fees from Apellis, AstraZeneca, Bayer, Blade Therapeutics, Boehringer Ingelheim, Bristol-Myers Squibb, 
Galapagos, GlaxoSmithKline R\&D, Indalo, Novartis, Pliant, ProMetic, Respivnat, Roche, Samumed and UCB. A.A. Chetta has nothing to disclose. P. Sestini has nothing to disclose. A.U. Wells reports lecture fees and advisory board fees from Boeringher Ingelheim, Roche and Bayer, outside the submitted work. E.A. Renzoni reports lecture fees and advisory board fees from Boeringher Ingelheim and Roche, and lecture fees from Mundipharma, outside the submitted work.

\section{References}

1 Nishiyama O, Taniguchi $\mathrm{H}$, Kondoh $\mathrm{Y}$, et al. Health-related quality of life in patients with idiopathic pulmonary fibrosis. What is the main contributing factor? Respir Med 2005; 99: 408-414.

2 Swigris JJ, Kuschner WG, Jacobs SS, et al. Health-related quality of life in patients with idiopathic pulmonary fibrosis: a systematic review. Thorax 2005; 60: 588-594.

3 Lancaster LH. Utility of the six-minute walk test in patients with idiopathic pulmonary fibrosis. Multidiscip Respir Med 2018; 13: 45.

4 Raghu G, Collard HR, Egan JJ, et al. An official ATS/ERS/JRS/ALAT statement: idiopathic pulmonary fibrosis: evidence-based guidelines for diagnosis and management. Am J Respir Crit Care Med 2011; 183: 788-824.

5 Lama VN, Flaherty KR, Toews GB, et al. Prognostic value of desaturation during a 6-minute walk test in idiopathic interstitial pneumonia. Am J Respir Crit Care Med 2003; 168: 1084-1090.

6 Visca D, Mori L, Tsipouri V, et al. Effect of ambulatory oxygen on quality of life for patients with fibrotic lung disease (AmbOx): a prospective, open-label, mixed-method, crossover randomised controlled trial. Lancet Respir Med 2018; 6: 759-770

7 Du Plessis JP, Fernandes S, Jamal R, et al. Exertional hypoxemia is more severe in fibrotic interstitial lung disease than in COPD. Respirology 2018; 23: 392-398.

8 Chetta A, Aiello M, Foresi A, et al. Relationship between outcome measures of six-minute walk test and baseline lung function in patients with interstitial lung disease. Sarcoidosis Vasc Diffuse Lung Dis 2001; 18: 170-175.

9 Wells AU, Desai SR, Rubens MB, et al. Idiopathic pulmonary fibrosis: a composite physiologic index derived from disease extent observed by computed tomography. Am J Respir Crit Care Med 2003; 167: 962-969.

10 Visca D, Tsipouri V, Mori L, et al. Ambulatory oxygen in fibrotic lung disease (AmbOx): study protocol for a randomised controlled trial. Trials 2017; 18: 201.

11 Healey JF. Statistics: A Tool for Social Research. 9th Edn. Belmont, Wadsworth, 2011.

12 Zisman DA, Karlamangla AS, Kawut SM, et al. Validation of a method to screen for pulmonary hypertension in advanced idiopathic pulmonary fibrosis. Chest 2008; 133: 640-645.

13 Wells A, Devaraj A, Renzoni EA, et al. Multidisciplinary evaluation in patients with lung disease associated with connective tissue disease. Semin Respir Crit Care Med 2019; 40: 184-193. 